\title{
Discrete element analysis of the excavation effect of cross-river tunnel on the surroundings
}

\author{
Dongyuan Liu ${ }^{1,2}$, Xiedong Zhang ${ }^{1}$ and Zhihua Zhang ${ }^{1}$ \\ ${ }^{1}$ School of Transportation, Wuhan University of Technology, Wuhan 430063, China \\ ${ }^{2}$ CCCC First Highway Engineering Co.,LTD, Beijing 100024, China
}

\begin{abstract}
Cross-river tunnel as one of the underground constructions is complicated during its construction. For stability of tunnel excavation, it is emergency to analyze the dynamic characteristics of tunnel deformation under high water pressure. Therefore, a cross-river tunnel model is proposed based on DEM in this paper. Stiffness of particles decreases during excavation process which is as one of excavation methods. Porosity ratio of original porosity over its value at different excavation time has been considered. Radial displacements of particles at different angle around the tunnel are detected during excavation process. It shows that large deformation occurs at the vault of the excavation zone which accompanies with large radial displacement. The upper half of the tunnel performs larger deformation than the lower half part which results in many cracks in the concrete lining, the high water pressure may play an crucial role in it.
\end{abstract}

\section{Introduction}

Substructures have been developed rapidly with the demand of transportation in recent decades. These substructures, such as basement parking, highway tunnels, metros and et al, play crucial roles in people's life. Designers and engineers have payed more attention to these constructions due to the high stress environment which may result in inconceivable disasters.

For the influence of tunnel excavation process on the surroundings, a large numbers of works have been conducted by scholars in the world. Yang[1] proposed an analytical model for the evolution of fractured zone in viscoelastic surrounding rock mass combining the sliding crack model and equivalent crack method, and the displacement after lining was set up is far more than the one at the moment of excavation just finished. Ding[2] summarized the safety risks and risk factors of the metro construction, interpreted the acquisition process of risk identification rules, and the production of rule-extensions and the storage of rule structures. Martino[3] conducted experimental analysis and showed that a zone of irreversibly damaged rock surrounding the excavation, which may include failed zones, inner and outer damage zones and a zone of excavation disturbance where the in situ stresses were influence by the excavation no damage was measurable.

For cross-river tunnel, the influence factors for tunnel safety, besides the above, the variation of water level should be taken into account. The tunnel excavation under high-water pressure has significant influence on the surrounding rock or soft clay, which may lead to collapse or piping. In view of past researches, however, little literature conducted by discrete element method exists on this subject.

In this paper, a large scale cross-river tunnel of two dimensional model is proposed based on discrete element method, which has large advantages in simulating large deformation and stress distribution in detail. Wuhan metro $2^{\text {nd }}$ line is as the case study. Excavation process has analyzed under high water pressure. The deformation and displacement of particles in different angles of the tunnel have been detected during excavation. The purpose of this paper is trying to reveal the dynamic characteristics of excavation effect on the surroundings when water pressure is taken into account.

\section{Discrete element method}

In the DEM, the interaction of the particles is treated as a dynamic process with states of equilibrium developing whenever the internal forces balance. The contact forces and displacements of a stressed assembly of particles are found by tracing the movements of the individual particles. Movements result from the propagation through the particle system of disturbances caused by specified wall and particle motion and/or body forces. This is a dynamic process in which the speed of propagation depends on the physical properties of the discrete system.

The calculations performed in the DEM alternate between the application of Newton's second law to the particles and a force-displacement law at the contacts[4], shown in Figure 1. Newton's second law is used to determine the motion of each particle arising from the contact and body forces acting upon it, while the force-displacement law is used to update the contact 
forces arising from the relative motion at each contact. The presence of walls in PFC2D requires only that the force-displacement law account for ball-wall contacts. Newton's second law is not applied to walls, since the wall motion is specified by the user.

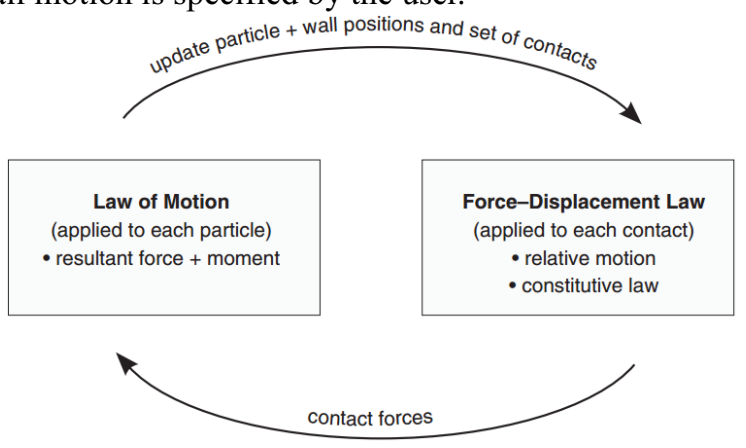

Figure 1. Calculation cycle in PFC.

The magnitude of the normal contact force is calculated by:

$$
F^{n}=K^{n} U^{n}
$$

where $K^{n}$ is the normal stiffness at the contact.

The overlap, $U^{n}$, defined to be the relative contact displacement in the normal direction, is given by:

$$
U^{n}=\left[\begin{array}{l}
R_{1}+R_{2}-d(\text { ball }- \text { ball }) \\
R_{1}-d(\text { ball }- \text { wall })
\end{array}\right.
$$

where $R_{1}, R_{2}$ are the radii of adjacent particles, respectively. $d$ is the distance between the ball centers.

The shear component of the contact displacement increment occurring over a timestep of $\Delta t$ is calculated by:

$$
\Delta U^{s}=V^{s} \Delta t
$$

and is used to calculate the shear elastic force increment as:

$$
\Delta F^{s}=-k^{s} \Delta U^{s}
$$

where $k^{s}$ is the shear stiffness at the contact.

\section{Cross-river tunnel model}

Alternating the three-dimensional method, the sample establishment, parameters of each strata and micromechanics of $2 \mathrm{D}$ numerical model of cross-river tunnel can be easily controlled. With the assumption of isotropic subsurface soils with finite thickness along the Yangtze River, the numerical model can be conducted through the direction view of the tunneling of STM. In order to analyze the influence of excavation process on cross-river tunnel, a cross-section surface of cross-river tunnel in Wuhan metro 2nd line is chosen to be the case study, the detail of the cross-section is shown in Figure 2. Since hydraulic coefficient of concrete lining is about $10^{-9}$ $\mathrm{m} / \mathrm{s}[5]$ and duration of maintenance for each time was relatively short, the effect of seepage on tunnel face stability is expected to be small before the concrete lining cracking. Thus, the effect of seepage is not taken into account in this study. As the silty clay being hard-solved problem in the world due to its low strength and strong plasticity[6], it is necessary to take silty clay into account in the model. Parameters of each stratum are shown in Table 1.

The excavation processes are as follows:

- Step 1. Deleting the particles in the range of the tunnel area.

- Step 2. Generating particles with smaller radii in the deleting area.

- Step 3. Decreasing particle stiffness gradually from the original to zero with thickness of concrete lining left.

Table 1. Macro parameters of each stratum in DEM modeling

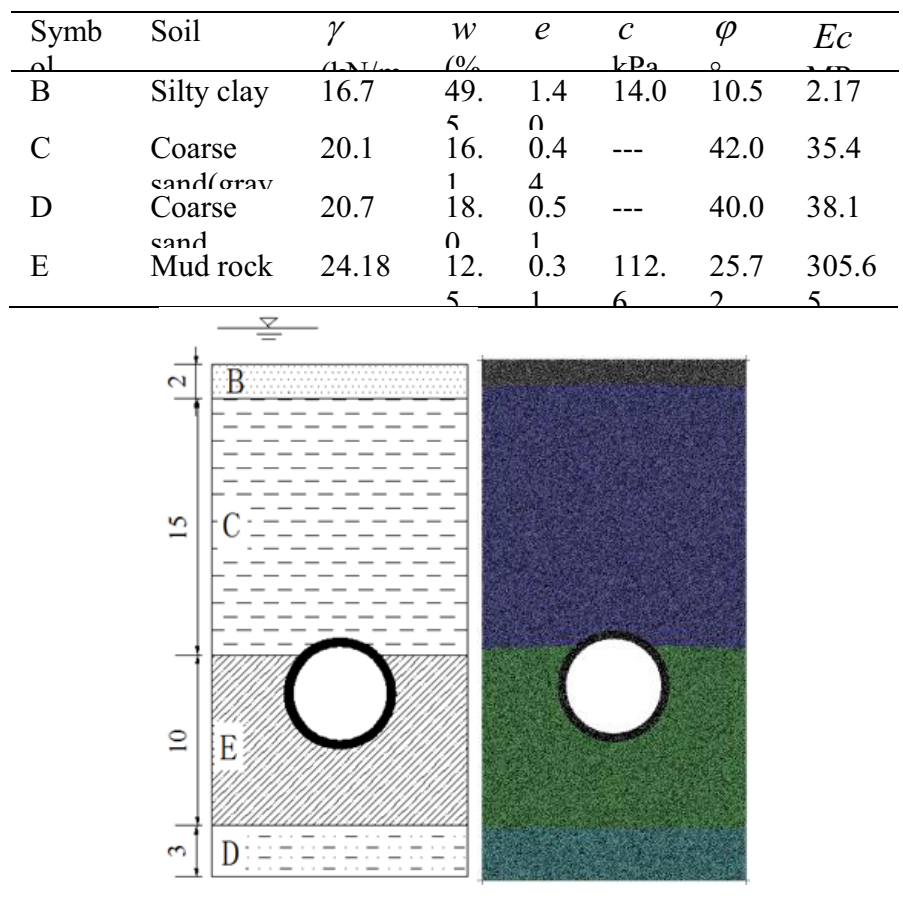

(a)

(b)

Figure 2. Cross-river tunnel model.(a): strata information of tunnel;(b) DEM model.

\section{Effect of excavation process on the surroundings}

\subsection{Porosity}

Many studies have been conducted around EDZ in formation and long-term processes[7] and area of damage[8]. In present model, the construction of lining would be completed in 2400 cycles. The variation of porosity of the surroundings at the distance of within 4.0 $\mathrm{m}$ has been tracked, the results are shown in Table 2 .

As shown in Table 2 that the porosity ratios of original porosity over its value of forthwith cycles are quite different after 2400 cycles and 22000 cycles were executed. Particles at the vault of the tunnel are 
compacted each other which the porosity ratio decrease as shown in Table 2 of angle $90^{\circ}$. The adjacent area at the upper half of the tunnel is compacting as well based on the data of porosity ratio of angle $45^{\circ}$ and $135^{\circ}$. The situation of deformation of the lower half of the tunnel in the surroundings is asymmetrical based on the data of angle $225^{\circ}$ and $315^{\circ}$. It is extremely visible to reveal the characteristics of porosity evolution that blue and red area represent surrounding material more loose and dense, respectively.

Table 2. Variation of porosity ratio between data at 2400 cycles and 22000 cycles

\begin{tabular}{cccc}
\hline $\begin{array}{c}\text { Distance from out edge of } \\
\text { lining }\end{array}$ & $0.1 \mathrm{~m}$ & $4.0 \mathrm{~m}$ \\
\hline & $0^{\circ}$ & $23.5 / 58.2$ & $-4.4 /-5.3$ \\
& $45^{\circ}$ & $-12.1 / 27.2$ & $-7.8 /-42.8$ \\
& $90^{\circ}$ & $7.6 /-12$ & $8.6 /-3.6$ \\
Porosity ratio (\%) & $135^{\circ}$ & $-16.3 /-24.6$ & $5.1 /-34.1$ \\
$\begin{array}{c}\text { (2400cycles/22000 } \\
\text { cycles) }\end{array}$ & $180^{\circ}$ & $-15.4 /-13.2$ & $5.2 /-16.1$ \\
& $225^{\circ}$ & $-3.8 / 5.7$ & $-7.9 /-3.4$ \\
& $270^{\circ}$ & $-7.4 / 4.6$ & $-1.1 / 0$ \\
& $315^{\circ}$ & $-16.4 /-10.4$ & $0.2 /-0.7$ \\
\hline
\end{tabular}

As shown in Table 2 that the porosity ratios of original porosity over its value of forthwith cycles are quite different after 2400 cycles and 22000 cycles were executed. Particles at the vault of the tunnel are compacted each other which the porosity ratio decrease as shown in Table 2 of angle $90^{\circ}$. The adjacent area at the upper half of the tunnel is compacting as well based on the data of porosity ratio of angle $45^{\circ}$ and $135^{\circ}$. The situation of deformation of the lower half of the tunnel in the surroundings is asymmetrical based on the data of angle $225^{\circ}$ and $315^{\circ}$. It is extremely visible to reveal the characteristics of porosity evolution that blue and red area represent surrounding material more loose and dense, respectively.

\subsection{Radial displacement}

The dependence of radial displacement on time around tunnel at distance from the lining outer edge of $0.5 \mathrm{~m}$ is depicted in Figure 3. As shown in Figure 3, after excavation, radial displacement grows as the iteration cycles increasing. The variation of radial displacements of angle $45^{\circ}, 90^{\circ}$ and $135^{\circ}$ are the largest in the surroundings. These points are located in the upper half circle around the tunnel, which is consistent with the characteristics of porosity and stress distribution. After 5000 cycles, radial displacement of the particle at the distance of outer edge of the concrete lining of $0.5 \mathrm{~m}$ at the other angles gradually tends to be constant. It should be noted that radial displacements of measurement points of $45^{\circ}, 90^{\circ}$ and $135^{\circ}$ at 22000 cycles increase.

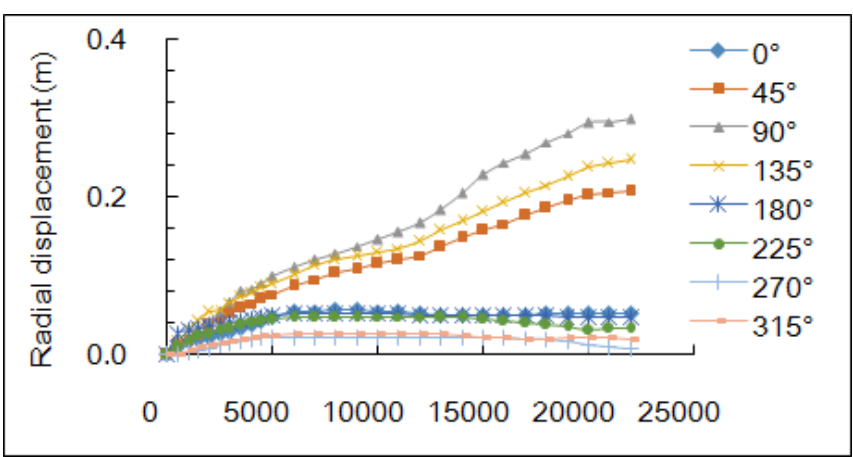

Figure 3. Radial displacement of the surroundings versus cycles at distance of $0.5 \mathrm{~m}$.

\subsection{Cracks tracking in the concrete lining}

Although the excavation disturbance zone problem has been conducted in many literatures, the information is mainly focus on the surrounding rock mass. However, as far as the crack weakened rock mass sensitive to the rock structure is considered, (which is sensitive to the rock structure,) the growth process of cracks should be taken into account, especially cracks occur in the concrete lining. As we known, concrete lining has large cohesive bond and high strength which results in low permeability, however, the infiltration effect will be imposed when cracks are generated in it. In present model, crack property has been tracked during excavation process, the illustration of crack distribution is shown in Figure 4.

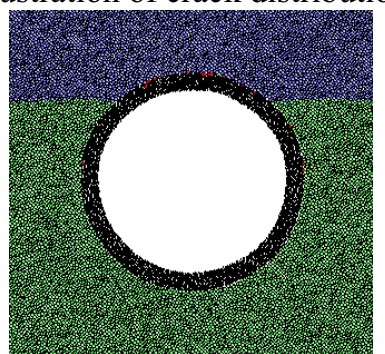

(a)

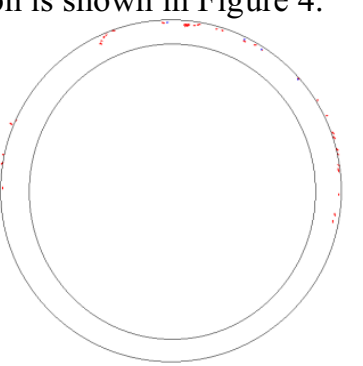

(b)
Figure 4. Cracks occur in the concrete lining after 22000 cycles.

As shown in Figure 4, cracks occur in the out edge of the concrete lining, it is remarkable to reveal the high stress of cross-river tunnel which may lead great strength of concrete lining to break. There are 55 cracks generated between particles when large compressive stress exceeds the bond strength as the process executing 22000 cycles. It is better understanding that cracks at the edge of the concrete lining have little influence on the stability of the tunnel, whereas the tunnel will be collapse or submerged when cracks running through the lining.

\section{Conclusions}

To investigate the influence of excavation process of cross-river tunnel on the surroundings, two dimensional discrete element model is conducted. As the excavation process executing with cycle increases, different variables of particles at different angles in the surroundings are detected. The following conclusions could be made: 
(1) During tunnel excavation, the vault of the tunnel in the surroundings is compressive as the porosity of local area decreases. Based on the data of porosity ratio in the surroundings, the deformation of the tunnel is not symmetrical along the center of the tunnel, which will lead the stress distribution of concrete lining to perform asymmetrical deformation.

(2) Based on radial displacement of particles around the excavation zone, large collapse occurs at the vault of the tunnel which is consistent with the data of porosity variation. Therefore, for stability of tunnel excavation, advanced reinforcement should be needed when the shield tunneling machine excavates the low-strength layer.

(3) Large deformation results in many cracks between particles, however, it is relative safe when the cracks just occur at the outer edge of the concrete lining.
1. H.Q. Yang, D. Huang, X.M. Yang, X.P. Zhou. Tunn. Undergr. Sp. Tech. 35: 78-88 (2013)

2. L.Y. Ding, H.L. Yu, H. Li, C. Zhou, X.G. Wu, M.H. Yu. Automat. Constr. 27: 120-137 (2012)

3. J.B. Martino, N.A. Chandler. Int. J. Rock Mech. Min. 41: 1413-1426 (2004)

4. Itasca Consulting Group. Inc. PFC2D, Version 4.0. Minneapolis: Itasca (2008)

5. F.L. Min, W. Zhu, X.R. Han, X.C. Zhong. Geotech. Spec. Publ. 204 GSP: 210-215 (2010)

6. Z.H. Zhang, X.D. Zhang, H.S. Qiu, M. Daddow. Constr. Build. Mater. 125: 564-573 (2016)

7. P. Blümling, F. Bernier, P. Lebon, C.D. Martin. Phys. Chem. Earth, Parts A/B/C. 32:588-599 (2007)

8. G. Bäckblom. Swedish Nuclear Fuel and Waste Management Co (2008)

\section{References}

